\title{
AN EXTENSION OF PELEG'S INEQUALITY
}

By

\author{
Juan C. Cesco \\ and
}

\section{Ezio Marchi}

IMA Preprint Series \# 2198

(April 2008)

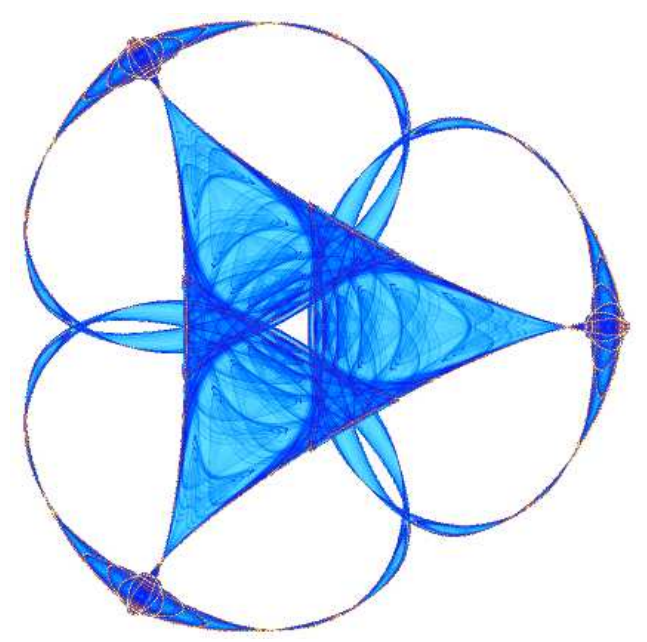

INSTITUTE FOR MATHEMATICS AND ITS APPLICATIONS

UNIVERSITY OF MINNESOTA 400 Lind Hall

207 Church Street S.E.

Minneapolis, Minnesota 55455-0436

Phone: 612-624-6066 Fax: 612-626-7370

URL: http://www.ima.umn.edu 


\title{
An extension of Peleg's inequality
}

by

Juan C. Cesco

Instituto de Matemática Aplicada San Luis (U.N. de San Luis-CONICET), ARGENTINA

(jcesco@unsl.edu.ar)

and

Ezio Marchi

Instituto de Matemática Aplicada San Luis (U.N. de San Luis-CONICET), ARGENTINA

emarchi@unsl.edu.ar

\begin{abstract}
In this note we prove an extension of a remarkable result due to B. Peleg. Peleg's result concerning with the simultaneous validity of a set of inequalities for families of functions defined on a finite product of standard simplices in finite dimensional spaces. The main result we prove here provides an extension of that result to the case of functions defined on a rather general product of simplices. Some topological requirements lead us to deal with this problem from a functional point of view.
\end{abstract}

\section{INTRODUCTION}

In Peleg (1967, lemma 2.4) a remarkable result is presented which is the main tool for proving several existence theorems concerning different solution concepts in cooperative game theory (Davis, Maschler (1965), Peleg (1967). In Cesco, Marchi (1990) Peleg's result is used to prove existence for a certain type of kernel and bargaining set in the framework of pure exchange economies.

Peleg's lemma concerns with the simultaneous validity of some inequalities for families of functions defined on a finite product of standard simplices in finite dimensional spaces. The main theorem in this paper provides an extension of that result to the case of functions defined on a rather general product of simplices. Some topological requirements leads us to deal with this problem from a functional point of view. We point out that we get a result which holds almost everywhere. In order to get a proof, we first prove an auxiliary result extending corollary 2.5 of Peleg (1967). That result has also been used extensively in game theory and has interest by itself. In fact, both Peleg's results, in the framework he worked, turn out to be equivalent.

This note is organized as follows. Definitions and notation are set forth in section 2. This section also includes an auxiliary result that we use later. The extension of Peleg's corollary is proved in section 3 and the main result is presented in the next one. We close with some final remarks.

\section{PRELIMINARIES.}


Let $N=\{1, \ldots, n\}$ and

$$
\Delta^{n-1}=\left\{\mathbf{x}=\left(x_{1}, \ldots, x_{n}\right) \in \mathbb{R}^{n}: \sum_{i \in N} x_{i}=1, x_{i} \geq 0, \text { for all } i \in N\right\}
$$

Here $\mathbb{R}$ denotes the set of real numbers and $\mathbb{R}^{n}$ the $n$-fold cartesian product of $\mathbb{R}$.

Let $\mathbb{T}=[0,1] \subseteq \mathbb{R}$ and let $L_{\infty}(\mathbb{T})$ be the space of all equivalence classes of measurable real functions defined on $\mathbb{T}$ endowed with the usual essential supremun norm $\|\cdot\|_{\infty}$. Here $\mu$ denotes the Lebesgue measure on $\mathbb{T}$.

Let

$$
\mathcal{X}(\mathbb{T})=\left\{f \in L_{\infty}(\mathbb{T}):\|f\|_{\infty} \leq 1\right\}
$$

Banach-Alaoglu's theorem asserts that $\mathcal{X}(\mathbb{T})$ is a weak ${ }^{*}$-compact subset of $L_{\infty}(\mathbb{T})$ whenever $L_{\infty}(\mathbb{T})$ is viewed as the dual space of $L_{1}(\mathbb{T})$. $\mathcal{X}(\mathbb{T})$ is also a locally convex metrizable space when it is endowed with the weak*-topology. We refer the reader to Rudin [13, Ch.3] for an account of those results.

Now let

$\mathcal{Y}(\mathbb{T})=\left\{\mathbf{f}=\left(f_{1}, \ldots, f_{n}\right): f_{i}: \mathbb{T} \rightarrow \mathbb{R}\right.$ is $\mu$-measurable for all $i \in N$ and $\left(f_{1}(t), \ldots, f_{n}(t)\right) \in \Delta^{n-1}$ a.e. $(\mu)$ in $\left.\mathbb{T}\right\}$

That set defines univocally, in a natural way, a subset $\mathcal{S}(\mathbb{T})$ of $\prod_{i \in N} \mathcal{X}(\mathbb{T})$, namely, that of all $n$-vectors of $\mu$-equivalence classes derived from the vectors of functions in $\mathcal{Y}(\mathbb{T})$.

By endowing $\prod_{i \in N} \mathcal{X}(\mathbb{T})$ with the product topology, which we are going to denote weak*-topology too, it turns to be that $\mathcal{S}(\mathbb{T})$ is a weak*-compact subset of $\prod_{i \in N} \mathcal{X}(\mathbb{T})$. Moreover, $\mathcal{S}(\mathbb{T})$ is a weak ${ }^{*}$-compact locally convex metrizable space.

Let

$$
C: \mathbb{T} \rightarrow 2^{\mathcal{S}(\mathbb{T})}
$$

be a mapping, namely, a set valued mapping from $\mathbb{T}$ into $\mathcal{S}(\mathbb{T})$.

We say that $C$ is measurable if

$$
\mathcal{C}=\{(t, \mathbf{f}) \in \mathbb{T} x \mathcal{S}(\mathbb{T}): \mathbf{f} \in \mathbf{C}(\mathbb{T})\}
$$

belongs to the product $\sigma$-field $\mathcal{L}(\mathbb{T}) \otimes \mathcal{B}(\mathcal{S}(\mathbb{T}))$. Here $\mathcal{L}(\mathbb{T})$ denotes the $\sigma$-field of all Lebesgue measurable subsets of $\mathbb{T}$ and $\mathcal{B}(\mathcal{S}(\mathbb{T}))$ the $\sigma$-field of all Borel subsets of $\mathcal{S}(\mathbb{T})$.

We close this section by stateing the following result (Castaing-Valadier $[2$, Theorem III.30]). 
Theorem 1: Let $(\mathbf{T}, \mathcal{L}, \nu)$ be a measure space with $\nu \geq 0 \sigma$-finite, $\mathcal{L}$ complete and $(\mathbf{S}, d)$ a complete separable metric space. Let $C: \mathbf{T} \rightarrow 2^{\mathbf{S}}$ a mapping that maps $\mathbf{T}$ into closed non empty subset of $\mathbf{S}$. Then, the following two properties are equivalent:

i) $C$ is measurable

ii) The real function

$$
\operatorname{dist}(C(.), f)=\inf \{d(g, f): f \in C(t)\}
$$

defined on $\mathbf{T}$, is measurable for all $f \in \mathbf{S}$.

\section{A GENERALIZATION OF PELEG'S COROLLARY.}

In this section we prove an auxiliary result, which, as we mentioned in the introduction, has great interest in its own.

Before stateing it we set the following convention. Given an element $\tilde{f}_{\infty} \in$ $L(\mathbb{T})$, which is an equivalence class, we said that a subset $\mathbb{F}$ of $\mathbb{T}$ is a support for $\tilde{f}$ if there exists a function $f$ belonging to the class $\tilde{f}$ such that $f(t)>0$ if and only if $t \in \mathbb{F}$. It follows clearly, from that definition that, if $\mathbb{F}^{\prime}$ and $\mathbb{F}^{\prime \prime}$ are two supports for a given class $\tilde{f}$, then $\mu\left(\mathbb{F}^{\prime} \Delta \mathbb{F}^{\prime \prime}\right)=0$. Here $\Delta$ indicates symmetric difference.

In what follows we assume that a particular support $\mathbb{F}$ has been chosen for each $\tilde{f} \in L_{\infty}(\mathbb{T})$. In the case of having $\tilde{\mathbf{f}}=\left(\tilde{f}_{1}, \ldots, \tilde{f}_{n}\right)$ in $\mathcal{S}(\mathbb{T}), \mathbb{F}_{i}$ will denote the chosen support for the class $\tilde{f}_{i}$.

Theorem 2: Let

$$
C^{i}: \mathbb{T} \rightarrow 2^{\mathcal{S}(\mathbb{T})} \quad i \in N
$$

be a family of non empty valued measurable mappings such that

i) $C^{i}(t)$ is weak ${ }^{*}$-closed for all $t \in \mathbb{T}$, and for all $i \in N$.

ii) $\bigcup_{j \in N} C^{j}(t)=\mathcal{S}(\mathbb{T}) a e(\mu)$.

iii) $C^{i}(t) \supseteq\left\{\tilde{\mathbf{f}} \in \mathcal{S}(\underset{\tilde{\mathbf{f}}}{\mathbb{T}}): t \notin \mathbb{F}_{i}\right\} \quad \forall i \in N, a e(\mu)$.

Then, there exists $\tilde{\mathbf{f}} \in \mathcal{S}(\mathbb{T})$ such that

$$
\tilde{\mathbf{f}} \in \mathbf{C}^{i}(t) \quad \forall i \in N, a e(\mu)
$$

Proof: We define the functions

$$
c^{i}: \mathbb{T} x \mathcal{S}(\mathbb{T}) \rightarrow \mathbb{R} \text { for all } i \in N
$$

by 


$$
\begin{aligned}
c^{i}(t, \tilde{\mathbf{f}}) & =\operatorname{dist}\left(C^{i}(t), \tilde{\mathbf{f}}\right) \\
& =\inf \left\{d^{*}(\tilde{\mathbf{g}}, \tilde{\mathbf{f}}): \tilde{\mathbf{g}} \in \mathbf{C}^{i}(t)\right\}
\end{aligned}
$$

where $d^{*}$ denotes a weak*-compatible metric in $\mathcal{S}(\mathbb{T})$.

It is clear that the functions

$$
c^{i}(t, .): \mathcal{S}(\mathbb{T}) \rightarrow \mathbb{R}
$$

are weak*-continuous for all $i \in N, t \in \mathbb{T}$.

On the other hand, we have that the real functions

$$
c^{i}(\tilde{\mathbf{f}}, .): \mathbb{T} \rightarrow \mathbb{R}
$$

are measurable for all $i \in N, \tilde{\mathbf{f}} \in \mathcal{S}$. This is so because of Theorem 1, that guarantees that the functions $d^{*}\left(C^{i}(),. \tilde{\mathbf{f}}\right)$ are measurable.

Now, for each $\tilde{\mathbf{f}} \in \mathcal{S}(\mathbb{T})$, let the real functions

$$
H_{i}(\tilde{\mathbf{f}}): \mathcal{S}(\mathbb{T}) \rightarrow \mathbb{R} \quad i \in N
$$

be defined by

$$
H_{i}(\tilde{\mathbf{f}})(t)=\frac{f_{i}(t)+c^{i+1}(t, \tilde{\mathbf{f}})}{1+\sum_{j \in N} c^{j}(t, \tilde{\mathbf{f}})} \quad \forall t \in \mathbb{T}
$$

where $\mathbf{f}=\left(f_{1}, \ldots, f_{n}\right)$ is an element of $\tilde{\mathbf{f}}$. Here we are adopting the convention that $n+1=1$. Clearly,

$$
0 \leq H_{i}(\tilde{\mathbf{f}})(t) \leq 1 a e(\mu)
$$

Besides, since $f_{i}$ and $c^{j}(., \tilde{\mathbf{f}}), j \in N$ are measurable functions, $H_{i}(\tilde{\mathbf{f}})$ is measurable too. Finally, we note that

$$
\sum_{j \in N} H_{j}(\tilde{\mathbf{f}})(t)=1 a e(\mu)
$$

From those considerations we conclude that the mapping

$$
\mathbf{H}: \mathcal{S}(\mathbb{T}) \rightarrow \mathcal{S}(\mathbb{T})
$$

defined by 


$$
\mathbf{H}(\tilde{\mathbf{f}})=\left(H_{1}(\tilde{\mathbf{f}}), \ldots, H_{n}(\tilde{\mathbf{f}})\right)
$$

is well defined.

Now, we are going to prove that $\mathbf{H}$ is a weak ${ }^{*}$-continuous mapping. For that, is enough to prove that each component $H_{i}$ is a weak*-continuous real function.

Let $\left\{\tilde{\mathbf{f}}^{r}\right\}_{r \geq 1}$ be a weak*-convergent sequence in $\mathcal{S}(\mathbb{T})$. That means that there exists $\tilde{\mathbf{f}} \in \mathcal{S}$ such that, for each $g \in L_{1}(\mathbb{T})$,

$$
\lim _{r} \int_{\mathbb{T}} f_{i}^{r}(t) g(t) d t=\int_{\mathbb{T}} f_{i}(t) g(t) d t
$$

Here $f_{i}^{r} \in \tilde{f}_{i}^{r}$. We have to show that

$$
\lim _{r} \int_{\mathbb{T}} H_{i}\left(\tilde{\mathbf{f}}^{r}\right)(t) \cdot g(t) d t=\int_{\mathbb{T}} H_{i}(\tilde{\mathbf{f}})(t) \cdot g(t) d t
$$

for all $g \in L_{1}(\mathbb{T})$. But

$$
\begin{aligned}
\int_{\mathbb{T}} H_{i}\left(\tilde{\mathbf{f}}^{r}\right)(t) \cdot g(t) d t= & \int_{\mathbb{T}} \frac{f_{i}^{\gamma}(t)}{1+\sum_{j \in N} c^{j}\left(t, \tilde{\mathbf{f}}^{\gamma}\right)} \cdot g(t) d t \\
& +\int_{\mathbb{T}} \frac{c^{i+1}\left(t, \tilde{\mathbf{f}}^{\gamma}\right)}{1+\sum_{j \in N} c^{j}\left(t, \tilde{\mathbf{f}}^{\gamma}\right)} \cdot g(t) d t
\end{aligned}
$$

We first note that

$$
\frac{c^{i+1}\left(t, \tilde{\mathbf{f}}^{\gamma}\right)}{1+\sum_{j \in N} c^{j}\left(t, \tilde{\mathbf{f}}^{\gamma}\right)} \cdot g(t) \leq g(t)
$$

for all $\gamma \geq 1, t \in \mathbb{T}$. Besides, because of the weak $^{*}$-continuity of the function $c^{j}(., t), j \in N, t \in \mathbb{T}$,

$$
\lim _{i} \frac{c^{i+1}\left(t, \tilde{\mathbf{f}}^{\gamma}\right)}{1+\sum_{j \in N} c^{j}\left(t, \tilde{\mathbf{f}}^{\gamma}\right)} \cdot g(t)=\frac{c^{i+1}(t, \tilde{\mathbf{f}})}{1+\sum_{j \in N} c^{j}(t, \tilde{\mathbf{f}})} \cdot g(t) \text { for all } t \in \mathbb{T}
$$

Hence, an application of Lebesgue's dominated convergence theorem yields that

$$
\lim _{i} \int_{\mathbb{T}} \frac{c^{i+1}\left(t, \tilde{\mathbf{f}}^{\gamma}\right)}{1+\sum_{j \in N} c^{j}\left(t, \tilde{\mathbf{f}}^{\gamma}\right)} \cdot g(t) d t=\int_{\mathbb{T}} \frac{c^{i+1}(t, \tilde{\mathbf{f}})}{1+\sum_{j \in N} c^{j}(t, \tilde{\mathbf{f}})} \cdot g(t)
$$

On the other hand, the inequality

$$
\left(1+\sum_{j \in N} c^{j}\left(\tilde{\mathbf{f}}^{r}\right)(t)\right) \cdot g(t) \leq \mathbf{k} \cdot g(t)
$$


is always true for a suitably chosen constant $\mathbf{k}$, for all $g \in L_{1}(\mathbb{T}), t \in \mathbb{T}$. This is so because the functions $c^{j}, j \in N$ are bounded since the distance $d^{*}$ is bounded on $\mathcal{S}$. This allows us to apply Lebesgue's theorem, as we did in the previous paragraph, to get that

$$
\lim _{i} \int_{\mathbb{T}}\left(1+\sum_{j \in N} c^{j}\left(\tilde{\mathbf{f}}^{r}\right)(t)\right) \cdot g(t) d t=\int_{\mathbb{T}}\left(1+\sum_{j \in N} c^{j}(\tilde{\mathbf{f}})(t)\right) \cdot g(t) d t
$$

for all $g \in L_{1}(\mathbb{T})$, namely, that the functions $1+\sum_{j \in N} c^{j}\left(\tilde{\mathbf{f}}^{r}\right)(t), \gamma \geq 1$ converge to $1+\sum_{j \in N} c^{j}(\mathbf{f})(t)$ in the weak* sense.

Now

$$
\begin{aligned}
& \mid \int_{\mathbb{T}} \frac{f_{i}^{\gamma}(t)}{1+\sum_{j \in N} c^{j}(t, \tilde{\mathbf{f}} \gamma)} \cdot g(t) d t-\int_{\mathbb{T}} \frac{f_{i}(t)}{1+\sum_{j \in N} c^{j}(t, \tilde{\mathbf{f}})} \cdot g(t) d t \\
& +\int_{\mathbb{T}} \frac{f_{i}(t)}{1+\sum_{j \in N} c^{j}\left(t, \tilde{\mathbf{f}}^{\gamma}\right)} \cdot g(t) d t-\int_{\mathbb{T}} \frac{f_{i}(t)}{1+\sum_{j \in N} c^{j}\left(t, \tilde{\mathbf{f}}^{\gamma}\right)} \cdot g(t) d t \mid \\
\leq & \int_{\mathbb{T}}\left|\frac{\left.f_{i}^{\gamma}(t)-f_{i}(t)\right)}{1+\sum_{j \in N} c^{j}\left(t, \tilde{\mathbf{f}}^{\gamma}\right)} \cdot g(t)\right| d t+\int_{\mathbb{T}}\left|f_{i}(t)\right|\left|\frac{1}{1+\sum_{j \in N} c^{j}(t, \tilde{\mathbf{f}} \gamma)}-\frac{1}{1+\sum_{j \in N} c^{j}\left(t, \tilde{\mathbf{f}}^{\gamma}\right)}\right| \cdot|g(t)| d t \\
\leq & \int_{\mathbb{T}}\left|\frac{\left.f_{i}^{\gamma}(t)-f_{i}(t)\right)}{1+\sum_{j \in N} c^{j}\left(t, \tilde{\mathbf{f}}^{\gamma}\right)}\right| \cdot|g(t)| d t \\
& +\left\|f_{i}\right\|_{\infty} \cdot \int_{\mathbb{T}}\left|\frac{1}{1+\sum_{j \in N} c^{j}(t, \tilde{\mathbf{f}} \gamma)}-\frac{1}{1+\sum_{j \in N} c^{j}(t, \tilde{\mathbf{f}} \gamma)}\right| \cdot|g(t)| d t
\end{aligned}
$$

Since $\left|\frac{1}{1+\sum_{j \in N} c^{j}(t, \tilde{\mathbf{f}} \gamma)}\right| \cdot|g(t)| \leq|g(t)|$ for all $\gamma \geq 1$, and taking into account the fact that $\lim _{\gamma}\left(f_{i}^{\gamma}(t)-f_{i}(t)\right)=0$, we can apply Lebesgue's theorem once more to get that the first term in the last member in the above chain of inequalities can be made arbitrarily small provided $\gamma$ is large enough.

Finally, since the function $\frac{g(t)}{1+\sum_{j \in N} c^{j}(t, \tilde{\mathbf{f}})}$ also belongs to $L_{1}(\mathbb{T})$ provided $g$ belongs to $L_{1}(\mathbb{T})$, and due to the fact that the sequence $\left\{f_{i}^{r}\right\}_{r \geq 1}$ converges to $f_{i}$ in the weak* sense, the second term in the aforementioned chain of inequalities can also be made arbitraily small if $\gamma$ is large enough. This proves that

$$
\lim _{i} \int_{\mathbb{T}} \frac{f_{i}^{\gamma}(t)}{1+\sum_{j \in N} c^{j}(t, \tilde{\mathbf{f}} \gamma)} \cdot g(t) d t=\int_{\mathbb{T}} \frac{f_{i}(t)}{1+\sum_{j \in N} c^{j}(t, \tilde{\mathbf{f}})} \cdot g(t) d t
$$

Combining (1) and (2) we obtain that the sequence $\left\{H_{i}\left(\tilde{\mathbf{f}}^{r}\right)\right\}_{r \geq 1}$ converges to $H_{i}(\tilde{\mathbf{f}})$ in the weak ${ }^{*}$ sense. 
Now we are able to apply Theorem 1 to the mapping $\mathbf{H}^{1}$ which yields a class $\tilde{\mathbf{f}} \in \mathcal{S}(\mathbb{T})$ such that $\mathbf{H}(\tilde{\mathbf{f}})=\tilde{\mathbf{f}}$. This equality implies that

$$
f_{i}(t)=\frac{f_{i}(t)+c^{i+1}\left(t, \tilde{\mathbf{f}}^{\gamma}\right)}{1+\sum_{j \in N} c^{j}\left(t, \tilde{\mathbf{f}}^{\gamma}\right)} \quad a e(\mu)
$$

for all $i \in N$. Namely, that there exists $\mathbb{A}_{i} \subseteq \mathbb{T}, \mu\left(\mathbb{A}_{i}\right)=0$ such that the equality (3) holds for all $t \in \mathbb{T}-\mathbb{A}_{i}$. Let us denote by $\mathbb{A}=\bigcup_{j \in N} \mathbb{A}_{j}$. Clearly, $\mu(\mathbb{A})=0$.

Now, let $\mathbb{B}=\left\{t \in \mathbb{T}: \sum_{j \in N} f_{j}(t) \neq 1\right\}$. The definition of $\mathcal{S}(\mathbb{T})$ yields that $\mu(\mathbb{B})=0$ too.

Let $\mathbb{C}=\left\{t \in \mathbb{T}: \bigcup_{\in N} C^{j}(t) \neq \mathcal{S}(\mathbb{T})\right\}$. The measure of $\mathbb{C}$ is zero too.

For each $j \in N$, let $\mathbb{D}_{j}=\left\{t \in \mathbb{T}: C^{i}(t) \supseteq\left\{\tilde{\mathbf{f}} \in \mathcal{S}(\mathbb{T}): t \notin \mathbb{F}_{i}\right\}\right.$. Then, because of $i i i), \mu\left(\mathbb{D}_{j}\right)=0$. Let us call $\mathbb{D}=\bigcup_{j \in N} \mathbb{D}_{j}$. Therefore, $\mu(\mathbb{D})=0$.

Finally, for each $j \in N$, let $\mathbb{G}_{j}=\mathbb{F}_{j} \cap\left\{t \in \mathbb{T}: f_{j}=0\right\}$. Then, $\mu\left(\mathbb{G}_{j}\right)=0$. Let $\mathbb{G}=\bigcup_{j \in N} \mathbb{G}_{j}$. Obviously, $\mu(\mathbb{G})=0$.

We claim that, for each $t \in \mathbb{T}-(\mathbb{A} \cup \mathbb{B} \cup \mathbb{C} \cup \mathbb{D} \cup \mathbb{G})$, there exists $i \in N$ such that

$$
t \in \mathbb{F}_{i} \text { and } \tilde{\mathbf{f}} \in \mathbf{C}^{i+1}(t)
$$

Clearly, due to $i i)$, that assertion is true if $\left\{j \in N: t \in \mathbb{F}_{j}\right\}=N$.

Otherwise, let $i$ be the greatest index such that $t \in \mathbb{F}_{i}$. Hence, because of iii) $\tilde{\mathbf{f}} \in \mathbf{C}^{i+1}(t)$ (Recall that $\left.n+1 \equiv 1\right)$.

We now point out that, $t \in \mathbb{F}_{i}-(\mathbb{A} \cup \mathbb{B} \cup \mathbb{C} \cup \mathbb{D} \cup \mathbb{G})$ implies that $f_{i}(t)>0$. Besides, $\tilde{\mathbf{f}} \in \mathbf{C}^{i+1}(t)$ implies that $c^{i+1}(t, \tilde{\mathbf{f}})=0$. These facts force that, for an index i satisfying (4), the equality (3) can take place if and only if $\sum_{j \in N} c^{j}(t, \tilde{\mathbf{f}})=0$. But this equality implies that $c^{j}(t, \tilde{\mathbf{f}})=0$ for all $j \in N$, which in turns yield that $\tilde{\mathbf{f}} \in \mathbf{C}^{j}(t)$ for all $j \in \mathrm{N}$. Since this property holds for all $t \in \mathbb{T}-(\mathbb{A} \cup \mathbb{B} \cup \mathbb{C} \cup \mathbb{D} \cup \mathbb{G})$ and for all $j \in N$, the theorem is proved.

\section{MAIN THEOREM.}

In this section we prove the main result of this note that is a simple consequence of theorem 2 .

Theorem 3: Let

$$
\begin{array}{ll}
g^{i} & : \quad \mathbb{T} x \mathcal{S}(\mathbb{T}) \rightarrow \mathbb{R} \\
h^{i} & : \quad \mathbb{T} x \mathcal{S}(\mathbb{T}) \rightarrow \mathbb{R}
\end{array}
$$

$i \in N$, be two families of weak ${ }^{*}$-continuous functions satisfying

i) $\forall(t, \tilde{\mathbf{f}}) \in \mathbb{T} x \mathcal{S}(\mathbb{T})$ there exists $i \in N$ such that $g^{i}(t, \tilde{\mathbf{f}}) \geq h^{i}(t, \tilde{\mathbf{f}})$.

ii) $h^{i}(t, \tilde{\mathbf{f}}) \leq \min \left\{g^{i}\left(t^{\prime}, \tilde{\mathbf{f}}^{\prime}\right):\left(t^{\prime}, \tilde{\mathbf{f}}\right) \in \mathbb{T} x \mathcal{S}(\mathbb{T})\right\}$ for all $(t, \tilde{\mathbf{f}}) \in \mathbb{T} x \mathcal{S}(\mathbb{T})$ such that $t \notin \mathbb{F}_{i}$. 
Then, there exists $\tilde{\mathbf{f}} \in \mathcal{S}(\mathbb{T})$ such that

$$
g^{j}(t, \tilde{\mathbf{f}}) \geq h^{j}(t, \tilde{\mathbf{f}}) \quad \forall j \in N, a e(\mu)
$$

As before, $\mathbb{F}_{i}$ denotes a chosen support for the i-th component of $\tilde{\mathbf{f}}=\left(\tilde{f}_{1}, \ldots, \tilde{f}_{n}\right)$. Proof:

Let $i \in N$ and

$$
C^{i}: \mathbb{T} \rightarrow 2^{\mathcal{S}(\mathbb{T})}
$$

be the mapping defined by

$$
C^{i}(t)=\left\{\tilde{\mathbf{f}} \in \mathcal{S}(\mathbb{T}): g^{i}(t, \tilde{\mathbf{f}}) \geq h^{i}(t, \tilde{\mathbf{f}})\right\}
$$

Because of the weak*-continuity of the functions $g^{i}$ and $h^{i}$ (and hence, of the functions $g^{i}(t,$.$\left.) and h^{i}(t,.) \forall t \in \mathbb{T}\right)$ and the compacity of the set $\mathcal{S}(\mathbb{T})$, the set $C^{i}(t)$ is non empty and weak*-closed for each $t \in \mathbb{T}$, for each $i \in \mathrm{N}$. Moreover, the weak* - continuty of $g^{i}$ and $h^{i}$ yields that

$$
\mathcal{C}^{i}=\left\{(t, \tilde{\mathbf{f}}) \in \mathbb{T} x \mathcal{S}(\mathbb{T}): g^{i}(t, \tilde{\mathbf{f}}) \geq h^{i}(t, \tilde{\mathbf{f}})\right\}
$$

is closed in $\mathbb{T} x \mathcal{S}(\mathbb{T})$ whenever this set is considered with the product topology $\mathcal{L}(\mathbb{T}) x \mathcal{B}(\mathcal{S}(\mathbb{T}))$. This proves that the mappings $C^{i}$ is measurable for each $i \in \mathrm{N}$.

On the other hand, because of $i$ ), given $t \in \mathbb{T}, \tilde{\mathbf{f}} \in \mathcal{S}(\mathbb{T})$, there exists $i \in N$ such that $\tilde{\mathbf{f}} \in \mathbf{C}^{i}(t)$. That proves that

$$
\bigcup_{j \in N} C^{j}(t)
$$

covers $\mathcal{S}(\mathbb{T})$ for each $t \in \mathbb{T}$.

Finally, if $t \notin \mathbb{F}_{i}$, because of $\left.i i\right), \tilde{\mathbf{f}} \in \mathbf{C}^{i}(t)$.

Those considerations show that the correspondences $C^{i}, i \in N$, satisfy the conditions $i$ ), $i i$ ) and $i i i$ ) of theorem 2 . Hence, that result guaratees the existence of $\tilde{\mathbf{f}} \in \mathcal{S}(\mathbb{T})$ such that

$$
\tilde{\mathbf{f}} \in \mathbf{C}^{j}(t) \quad \forall j \in N, a e(\mu)
$$

But, clearly, such an element satisfies that

$$
g^{j}(t, \tilde{\mathbf{f}}) \geq h^{i}(t, \tilde{\mathbf{f}}) \quad \forall j \in N, a e(\mu)
$$

which is (5). This completes the proof. 


\section{FINAL REMARKS.}

Our theorem 3 is a true generalization of Peleg's Lemma which is obtained by considering $h^{i}(t, \tilde{\mathbf{f}})=f_{i}(t)$, in a context that take $\mathbb{T}$ finite. In the general case, however, this particular form for the h's function cannot be adopted since they fail to be weak*-continuous.

We also note that ii) can be stated in Peleg's terms by using nonnegative functions.

We would like to point out that in [4], the authors proved an extension of another result due to Peleg ([11] lemma) which in turns is a generalization of the wellknown Kaster, Kurarowski and Mazurkiewicz lemma, similar to theorem 3 in this note. That result was obtained for every $t \in \mathbb{T}$. However, the conditions put on the multivalued mappings involved are stronger. We require that the multivalued mappings are upper and lower weak*-semicontinuous.

We close by mentioning that theorem 3 as well as theorem 2 can be used as tools for proving existence theorems for equilibrium concepts defined in a game theoretic framework considering a measure space of players.

\section{BIBLIOGRAPHY.}

[1] Browder, F., The fixed point theory of multi-valued mappings in topological vector spaces, Math. Annalen 177, 1968, 283-301.

[2] Castaing, C., and Valadier, M., Convex analysis and measurable multifunctions, Lecture notes in Mathematics, 480, Springer Verlag, 1977.

[3] Cesco,J. and Marchi, E., A general kernel type solution for pure exchange economies, Revista de Matemáticas Aplicadas 11, 1990, 29,36.

[4] Cesco, J. and Marchi, E., KKM in an arbitrary product of simplices, Preprint, IMASL, San Luis, 1990.

[5] Davis, M. and Maschler, M., The Kernel of a cooperative game, Naval, Res. Logist. Quart. 12, 1965, 223,259.

[6] Fan, K., Fixed points and minimax theorems in locally convex linear spaces, Proc. Nat.Acad. Sci. (USA) 38, 1952, 121-126.

[7] Fan, K., A minimax inequality and applications, in Inequalities III, Ed. Shisha, O., Academic Press, N.Y., 1972, 103-113.

[8] Glicksberg, L., A further generalization of the Kakutani's fixed point theorem with applications to Nash equilibrium points, Proc. Amer. Math. Soc. 3, 1953, 170-174.

[9] Knaster, B., Kuratowski, K. and Mazurkiewicz, S., Ein Beweis des fixpunktsatze fur $n$-dimensionale simplexe, Fundamenta Mathematica 14, 1929, 132-137.

[10] Marchi, E. and Martinez Legaz, J., A generalization of Browder's fixed point theorem and its applications, Preprint, Universitat de Barcelona, Spain, 1990.

[11] Peleg, B., Existence theorem for the bargaining set $\mathcal{M}_{1}^{(i)}$, in Essays in Mathematical Economics, Ed. M. Shubik, Chapter 4, Princeton University Press, 1967.

[12] Peleg, B., Equilibrium points for open acyclic relations, Canadian J. Math. 19, 1967, 366-369. 
[13] Rudin, W., Functional Analysis, MacGraw Hill, 1973. 